\title{
Erratum: In vitro studies of the ablation mechanism of periodontopathic bacteria and decontamination effect on periodontally diseased root surfaces by erbium: yttrium-aluminum-garnet laser
}

\author{
Fumihiko Akiyama • Akira Aoki • Mako Miura-Uchiyama • Katia M. Sasaki • \\ Shizuko Ichinose • Makoto Umeda • Isao Ishikawa • Yuichi Izumi
}

Published online: 18 December 2010

(C) Springer-Verlag London Ltd 2010

\section{Erratum to: Lasers Med Sci \\ DOI 10.1007/s10103-010-0763-3}

The original version of this article, unfortunately, contained several mistakes.

In page 1, the article's corresponding authors are Fumihiko Akiyama and Akira Aoki. The correct e-mail address for Doctor Akiyama is fumihiko-akiyama@waltz. ocn.ne.jp.

Figure 1 legend should be:

Fig. 1 Outline of experiments 1 and 2 (US: ultrasonic scaler)

The online version of the original article can be found at http://dx.doi. org/10.1007/s10103-010-0763-3.

\footnotetext{
F. Akiyama $(\bowtie) \cdot$ A. Aoki $(\bowtie) \cdot$ M. Miura-Uchiyama

M. Umeda $\cdot$ Y. Izumi

Periodontology Section, Department of Hard Tissue Engineering,

Graduate School, Tokyo Medical and Dental University,

1-5-45, Yushima,

Bunkyo-ku, Tokyo 113-8549, Japan

e-mail: fumihiko-akiyama@waltz.ocn.ne.jp
}

\section{A. Aoki \\ e-mail: aoperi@tmd.ac.jp}

K. M. Sasaki

Department of Dentistry, Brasilia University,

Brasilia, Brazil
For the last paragraph of SEM Observation, the citation for figure 5 should be (Fig. 5e,f) instead of (Fig. 5f)e,f.

Under Discussion, the first sentence of the third paragraph should be:

On the bactericidal effect of the Er:YAG laser, Schoop et al. compared the bactericidal effects of the Nd:YAG, diode, Er:YAG, and erbium, chromium:yttrium-scandium-gallium-garnet (Er,Cr:YSGG) lasers under standardized conditions from an endodontic aspect and reported that the Er: YAG laser showed the best bactericidal effect [34].

In Reference 38 "Bacteroides" should be "Black-pigmented Bacteroides".

\footnotetext{
S. Ichinose

Instrumental Analysis Research Center,

Tokyo Medical and Dental University,

Tokyo, Japan

I. Ishikawa

Institute of Advanced Biomedical Engineering and Science,

Tokyo Women's Medical University,

Tokyo, Japan

Y. Izumi

Global Center of Excellence Program, International Research

Center for Molecular Science in Tooth and Bone Diseases,

Tokyo Medical and Dental University,

Tokyo, Japan
} 